Supporting Information

\title{
Electrochemical Generation of Single Emulsion Droplets and In Situ Observation of Collisions on an Ultramicroelectrode
}

\author{
Sangmee Park, Hyunju Kim, Junghyun Chae*, and Jinho Chang* \\ Department of Chemistry and Center for NanoBio Applied Technology, Sungshin Women’s \\ University, 55 Dobong-ro 76 ga-gil, Gangbuk-gu, Seoul 142-732, Korea
}




\section{Table of Contents}

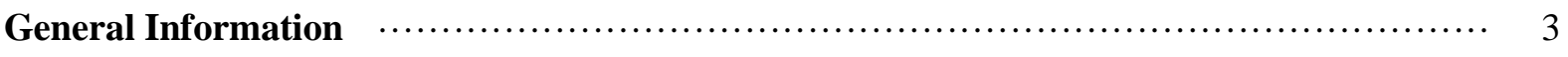

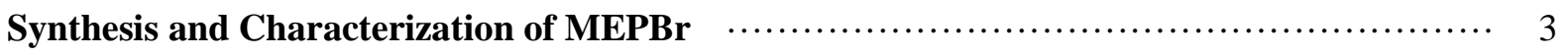

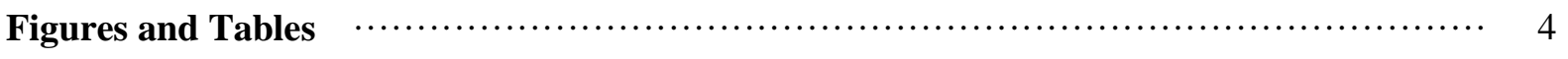

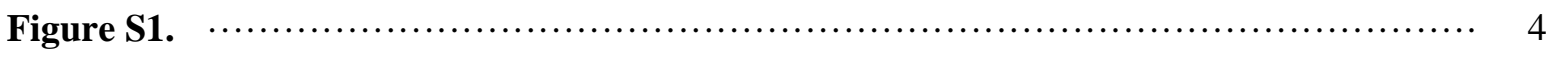

${ }^{1} \mathrm{H}-\mathrm{NMR}$ and ${ }^{13} \mathrm{C}$ - NMR of MEPBr

Figure S2.

(a) $0.5 \mathrm{M} \mathrm{H}_{2} \mathrm{SO}_{4}$ aqueous solution containing $100 \mathrm{mM} \mathrm{MEPBr}$. (b) and (c) solution after injection of $100 \mathrm{mM} \mathrm{Br} 2$; chemically formed, brownish oily droplets of $\mathrm{MEPBr}_{3}$ were shown after injection of $\mathrm{Br}_{2}$ into the $\mathrm{MEPBr}$ aqueous solution.

Figure S3.

CVs measured in $0.5 \mathrm{M} \mathrm{H}_{2} \mathrm{SO}_{4}$ with (black) / without (blue) $32 \mathrm{mM} \mathrm{MEPBr}$ on Pt UME at $0.02 \mathrm{~V} / \mathrm{s}$.

Figure S4.

Frequency counts vs. $i_{\text {spike }}$ obtained from CAs measured in different $E_{\text {ox }}$

Figure S5.

CAs measured in aqueous MEPBr solutions $\left(C_{M E P B r}(a q)=32.8,37.5,52,72,92 \mathrm{mM}\right)$ and frequency counts vs. $i_{\text {spike }}$ obtained from the CAs.

Figure S6.

Eight different current spikes (black line) with different $i_{\text {spike }}$ attributed to the electro-oxidation reaction of $\mathrm{Br}^{-}$in single $\mathrm{MEPBr}_{3}$ droplets from a CA in $C_{M E P B r(a q)}=72 \mathrm{mM}$, and their corresponding theoretical curves (blue triangle) derived from the bulk electrolysis model.

Table S1.

Estimated average $r_{\mathrm{e}}$ and $C_{\mathrm{Br}^{-}\left(\mathrm{MEPBr}_{3}\right)}$ by fitting current transients associated with $\mathrm{Br}^{-}$electrooxidation in single $\mathrm{MEPBr}_{3}$ droplets into the bulk electrolysis model shown in Figure S7. 


\section{General Information}

Chemicals: All solutions are prepared with deionized Milli-Q water. Sodium bromide $(\mathrm{NaBr}$, $>99 \%)$ and surfuric acid $\left(\mathrm{H}_{2} \mathrm{SO}_{4}, 98 \%\right)$ were purchased from Daejung Chemicals (Cheongwon, Korea). $N$-Methyl pyrrolidine $\left(\mathrm{C}_{5} \mathrm{H}_{11} \mathrm{~N}_{1},>98 \%\right)$ and ethyl bromide $\left(\mathrm{CH}_{3} \mathrm{CH}_{2} \mathrm{Br},>99 \%\right)$ were purchased from TCI Korea. Ethyl acetate was purchase from Duksan chemicals (Ansan, Korea). All reagents and solvents were used without further purification.

Instruments and measurements: ${ }^{1} \mathrm{H}$ and ${ }^{13} \mathrm{C}$ NMR spectra were recorded on a $500 \mathrm{MHz}$ spectrometer (Varian VNMRS 500) and LC-MS spectra were recorded using ESI mode (Hewlett Packard Series 1100 and Agilent Technologies 6130). CHI-600e potentiostat (CH Instruments, Austin, TX) was used for all electrochemical measurements. Three electrodes were used in an electrochemical cell; Pt ultramicroelectrodes (radius: $a=5 \mu \mathrm{m}$ ) as working electrodes, $\mathrm{Ag} / \mathrm{AgCl}(1 \mathrm{M}$ $\mathrm{KCl})$ as a reference electrode, and Pt wire as a counter electrode. All electrodes were purchased and used from $\mathrm{CH}$ Instruments. The all aqueous solutions contain $0.5 \mathrm{M} \mathrm{H}_{2} \mathrm{SO}_{4}$ under the deaerated condition with Ar.

\section{Synthesis and Characterization of MEPBr ${ }^{1}$}<smiles>CC[N+]1(C)CCCC1</smiles>

(CAS No. 69227-51-6)

To a solution of 1-methylpyrrolidine $(8.5 \mathrm{~g}, 100 \mathrm{mmol})$ in ethyl acetate $(20 \mathrm{~mL})$ was added ethyl bromide $(9.2 \mathrm{~mL}, 120 \mathrm{mmol})$ dropwise in ice-bath. Then the mixture was allowed to stir at room temperature for $6 \mathrm{~h}$. The solid product was filtered, washed with ethyl acetate for 3 times and dried in vacuum to give the product as white solid (17.7 g, 91\%). ${ }^{1} \mathrm{H}$ NMR (500 MHz, DMSO) $\delta 1.26$ (t, $J=$ $7.2 \mathrm{~Hz}, 3 \mathrm{H}), 2.07$ (m, 4H), 2.95 (s, 3H), 3.35-3.52 (m, 6H); ${ }^{13} \mathrm{C}$ NMR (125 MHz, DMSO) $\delta 63.58$, 59.03, 47.59, 21.77, 9.58; MS (m/z) ES ${ }^{+} 114.2 ;$ ES $^{-} 79.2$.

\footnotetext{
${ }^{1}$ Gu, F.; Dong, H.; Li, Y.; Sun, Z.; Yan, F., Macromolecules, 2014, 47, 6740-6747.
} 


\section{Figures and Tables}

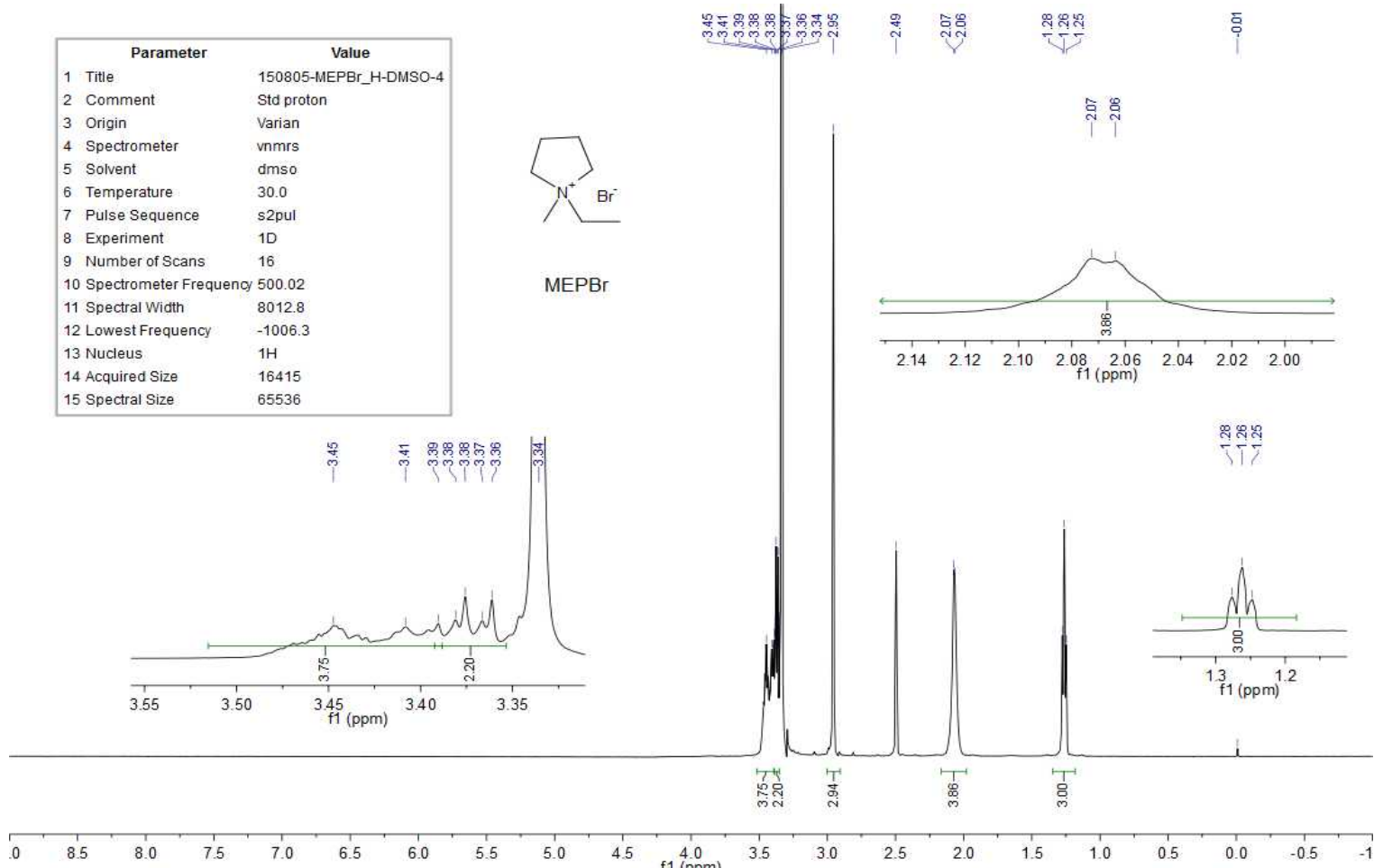

\begin{tabular}{|lll|}
\hline \multicolumn{1}{|c|}{ Parameter } & \multicolumn{1}{c|}{ Value } \\
1 & Title & 150805-MEPB__C-DMSO \\
2 & Comment & Std proton \\
3 & Origin & Varian \\
4 & Spectrometer & vnmrs \\
5 & Solvent & dmso \\
6 & Temperature & 30.0 \\
7 & Pulse Sequence & s2pul \\
8 & Experiment & 10 \\
9 & Number of Scans & 5512 \\
10 Spectrometer Frequency 125.74 \\
11 Spectral Width & 30487.8 \\
12 Lowest Frequency & -2042.4 \\
13 Nucleus & $13 \mathrm{C}$ \\
14 Acquired Size & 39649 \\
15 Spectral Size & 131072 \\
\hline
\end{tabular}

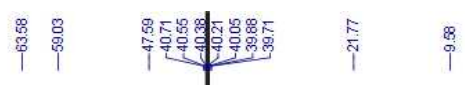

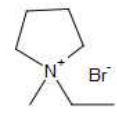

$\mathrm{MEPBr}$

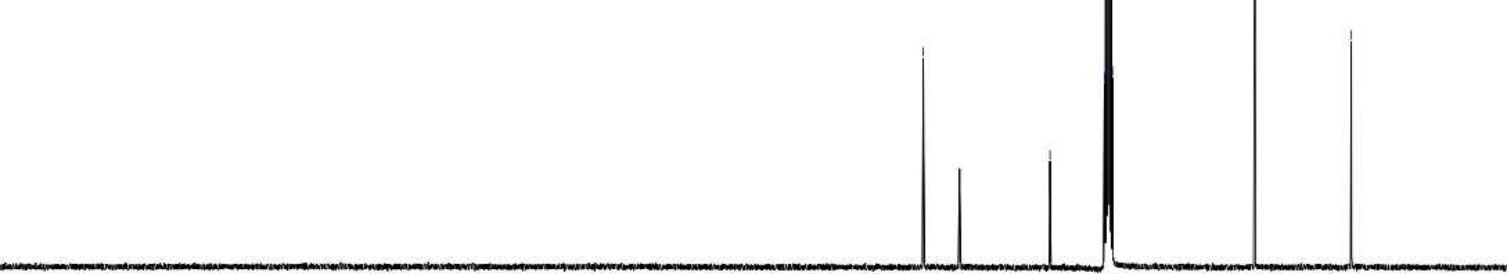

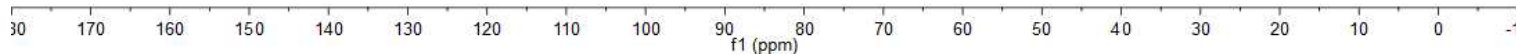

Figure S1. ${ }^{1} \mathrm{H}-\mathrm{NMR}$ and ${ }^{13} \mathrm{C}$-NMR of MEPBr 
Bulk $\mathrm{MEPBr}_{3}$ formation. The single $\mathrm{MEPBr}_{3}$ emulsion droplets, which are electrochemically generated on Pt UME were difficult to be observed since they are mainly distributed near to the $\mathrm{Pt}$ UME surface. In order to elucidate the formation of $\mathrm{MEPBr}_{3}$ and visualize the $\mathrm{MEPBr}_{3}$ droplets, we added $\mathrm{Br}_{2}$ into a $0.5 \mathrm{M} \mathrm{H}_{2} \mathrm{SO}_{4}$ aqueous solution containing MEPBr. Figure S2a shows a transparent $0.5 \mathrm{M} \mathrm{H}_{2} \mathrm{SO}_{4}$ solution with $100 \mathrm{mM} \mathrm{MEPBr}$. After the injection of $100 \mathrm{mM} \mathrm{Br}_{2}$ into the MEPBr solution, the color changed to be red and many $\mathrm{MEPBr}_{3}$ emulsion droplets were observed shown in Figure S2b and c. The size of the $\mathrm{MEPBr}_{3}$ emulsion droplets, which are electrochemically generated on Pt UME would be much smaller than the droplets shown in Figure S2 because electrochemical generation of $\mathrm{Br}_{3}{ }^{-}$are only limited to the UME surface.

(a)

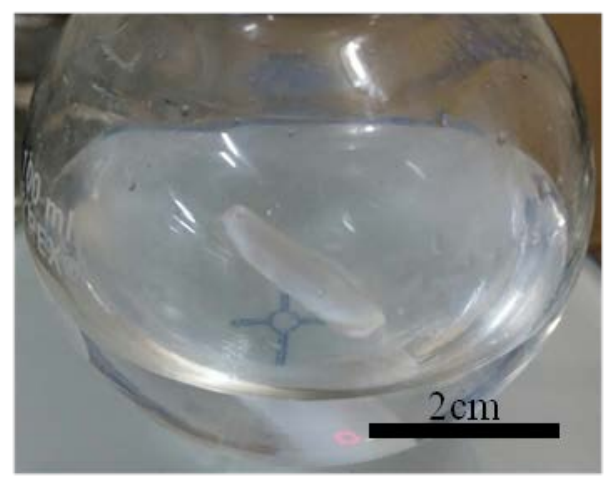

(b)

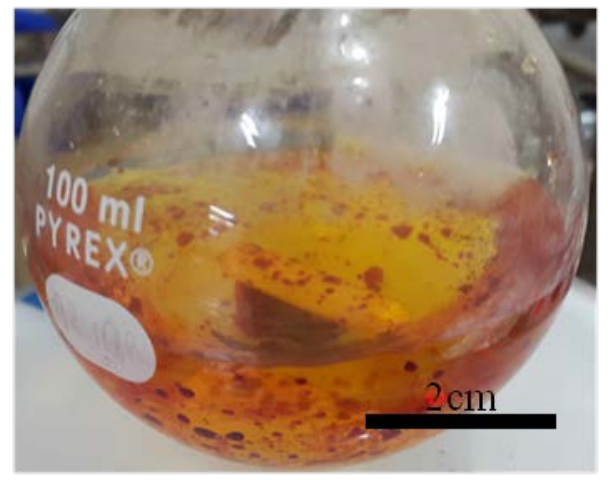

(c)

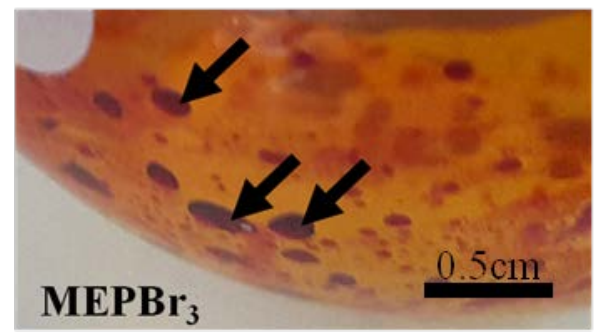

Figure S2. (a) $0.5 \mathrm{M} \mathrm{H}_{2} \mathrm{SO}_{4}$ aqueous solution containing $100 \mathrm{mM} \mathrm{MEPBr}$ (b) and (c) solution after injection of $100 \mathrm{mM} \mathrm{Br}_{2}$; chemically formed, brownish oily droplets of $\mathrm{MEPBr}_{3}$ were shown after injection of $\mathrm{Br}_{2}$ into the $\mathrm{MEPBr}$ aqueous solution. 


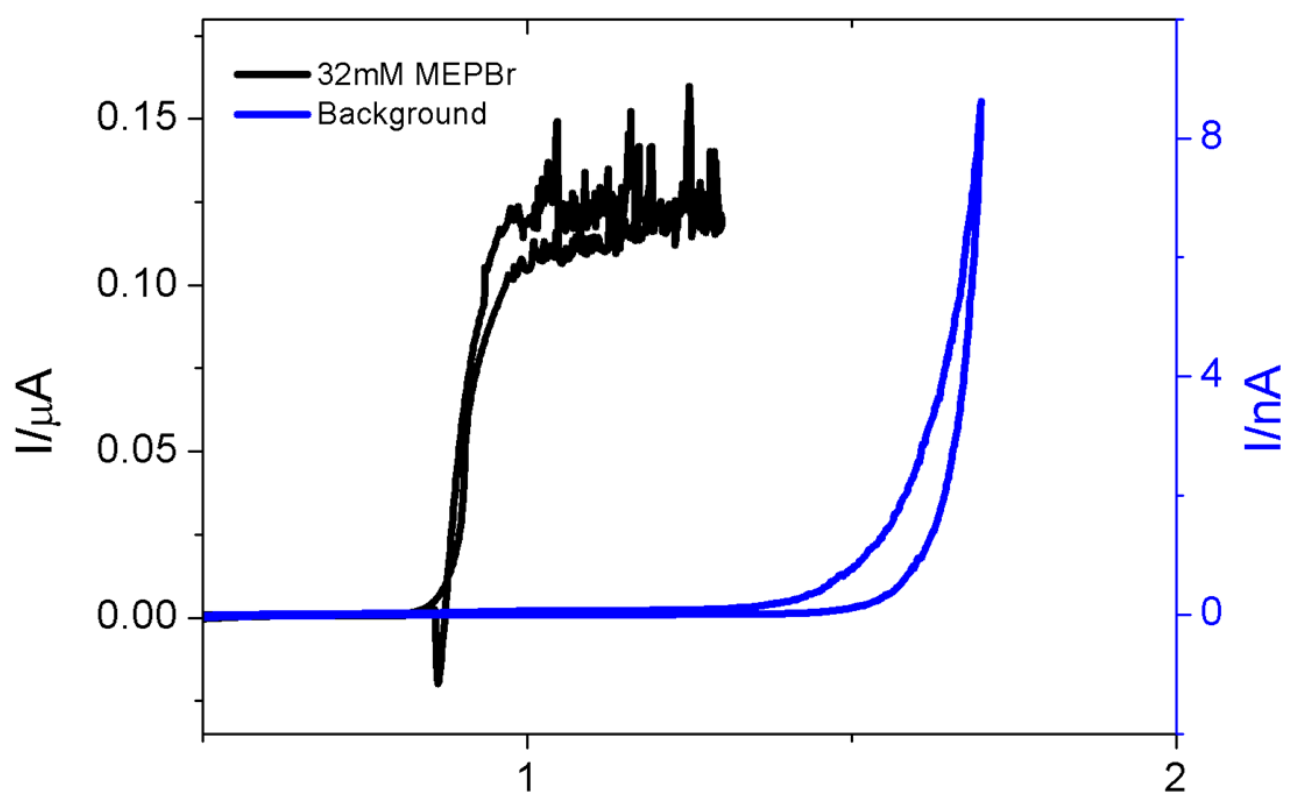

$\mathrm{E} / \mathrm{V}$ vs. $\mathrm{Ag} / \mathrm{AgCl}$

Figure S3. CVs measured in $0.5 \mathrm{M} \mathrm{H}_{2} \mathrm{SO}_{4}$ with (black) / without (blue) $32 \mathrm{mM} \mathrm{MEPBr}$ on Pt UME at $0.01 \mathrm{~V} / \mathrm{s}$. 

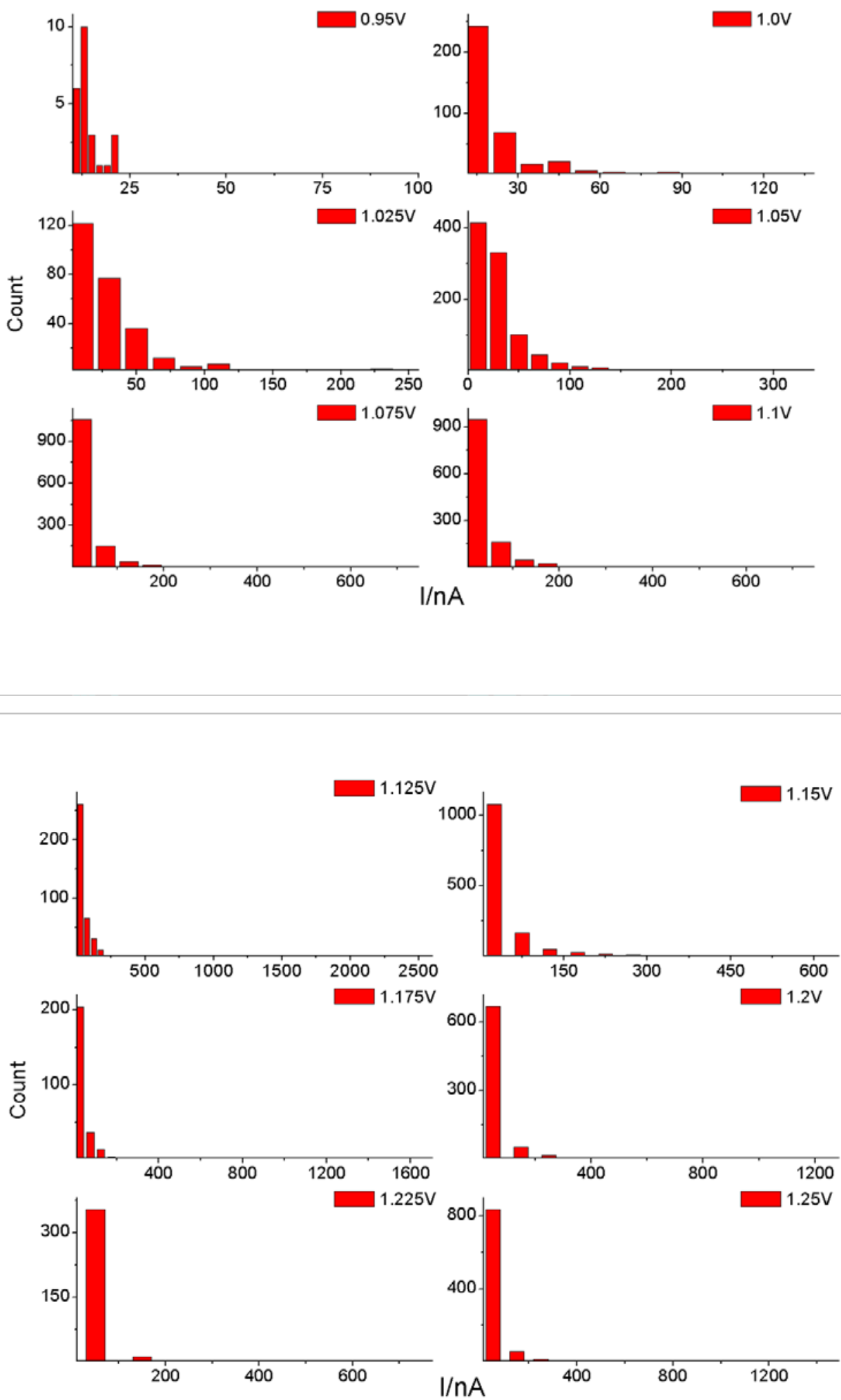


\begin{tabular}{|c|c|c|c|c|c|c|c|}
\hline \multicolumn{2}{|c|}{$E_{\mathrm{ox}}=0.95 \mathrm{~V}$} & \multicolumn{2}{|c|}{$E_{\mathrm{ox}}=1.0 \mathrm{~V}$} & \multicolumn{2}{|c|}{$E_{\mathrm{ox}}=1.025 \mathrm{~V}$} & \multicolumn{2}{|c|}{$E_{\mathrm{ox}}=1.05 \mathrm{~V}$} \\
\hline $\begin{array}{c}\text { Current } \\
\text { (nA) }\end{array}$ & $\begin{array}{c}i_{\text {spike }} \\
\text { counts }\end{array}$ & $\begin{array}{c}\text { Current } \\
\text { (nA) }\end{array}$ & $\begin{array}{c}i_{\text {spike }} \\
\text { counts }\end{array}$ & $\begin{array}{c}\text { Current } \\
\text { (nA) }\end{array}$ & $\begin{array}{c}i_{\text {spike }} \\
\text { counts }\end{array}$ & $\begin{array}{l}\text { Current } \\
\text { (nA) }\end{array}$ & $\begin{array}{c}i_{\text {spike }} \\
\text { counts }\end{array}$ \\
\hline 11 & 6 & 15 & 243 & 10 & 122 & 10 & 414 \\
\hline 13 & 10 & 25 & 69 & 30 & 77 & 30 & 331 \\
\hline 15 & 3 & 35 & 17 & 50 & 36 & 50 & 101 \\
\hline 17 & 1 & 45 & 21 & 70 & 12 & 70 & 45 \\
\hline 19 & 1 & 55 & 6 & 90 & 5 & 90 & 23 \\
\hline 21 & 3 & 65 & 4 & 110 & 7 & 110 & 12 \\
\hline & & 75 & 1 & 130 & 2 & 130 & 10 \\
\hline & & 85 & 4 & 150 & 1 & 150 & 2 \\
\hline & & 95 & 1 & 170 & 2 & 170 & 2 \\
\hline & & 105 & 0 & 190 & 0 & 190 & 1 \\
\hline & & 115 & 1 & 210 & 0 & 210 & 2 \\
\hline & & 125 & 1 & 230 & 3 & 230 & 1 \\
\hline & & & & & & 250 & 0 \\
\hline & & & & & & 270 & 1 \\
\hline & & & & & & 290 & 0 \\
\hline & & & & & & 310 & 0 \\
\hline & & & & & & 330 & 1 \\
\hline
\end{tabular}

\begin{tabular}{|cc|cc|cc|cc|}
\hline \multicolumn{2}{|c|}{$E_{\text {ox }}=1.075 \mathrm{~V}$} & \multicolumn{2}{c|}{$E_{\text {ox }}=1.1 \mathrm{~V}$} & \multicolumn{2}{c|}{$E_{\text {ox }}=1.125 \mathrm{~V}$} & \multicolumn{2}{c|}{$E_{\text {ox }}=1.15 \mathrm{~V}$} \\
\hline $\begin{array}{c}\text { Current } \\
\text { (nA) }\end{array}$ & $\begin{array}{c}i_{\text {spike }} \\
\text { counts }\end{array}$ & $\begin{array}{c}\text { Current } \\
(\mathrm{nA})\end{array}$ & $\begin{array}{c}i_{\text {spike }} \\
\text { counts }\end{array}$ & $\begin{array}{c}\text { Current } \\
(\mathrm{nA})\end{array}$ & $\begin{array}{c}i_{\text {spike }} \\
\text { counts }\end{array}$ & $\begin{array}{c}\text { Current } \\
(\mathrm{nA})\end{array}$ & $\begin{array}{c}i_{\text {spike }} \\
\text { counts }\end{array}$ \\
\hline 25 & 1055 & 25 & 946 & 25 & 261 & 25 & 1083 \\
75 & 150 & 75 & 162 & 75 & 66 & 75 & 164 \\
125 & 39 & 125 & 46 & 125 & 31 & 125 & 49 \\
175 & 16 & 175 & 26 & 175 & 12 & 175 & 26 \\
225 & 7 & 225 & 4 & 225 & 3 & 225 & 15 \\
275 & 4 & 275 & 2 & 275 & 1 & 275 & 9 \\
325 & 0 & 325 & 4 & 325 & 0 & 325 & 3 \\
375 & 2 & 375 & 0 & 375 & 1 & 375 & 1 \\
425 & 2 & 425 & 1 & 425 & 0 & 425 & 1 \\
475 & 0 & 475 & 2 & $\vdots$ & 0 & 475 & 2 \\
525 & 0 & 525 & 1 & 2575 & 1 & 525 & 1 \\
575 & 1 & 575 & 0 & & & 575 & 2 \\
625 & 0 & 625 & 0 & & & 625 & 0 \\
675 & 0 & 675 & 0 & & & & \\
725 & 1 & 725 & 1 & & & & \\
\hline$E_{\text {ox }}=1.175 \mathrm{~V}$ & \multicolumn{2}{|c|}{$E_{\text {ox }}=1.2 \mathrm{~V}$} & & & & \\
\hline
\end{tabular}




\begin{tabular}{|cc|cc|cc|cc|}
\hline $\begin{array}{c}\text { Current } \\
(\mathrm{nA})\end{array}$ & $\begin{array}{c}i_{\text {spike }} \\
\text { counts }\end{array}$ & $\begin{array}{c}\text { Current } \\
(\mathrm{nA})\end{array}$ & $\begin{array}{c}i_{\text {spike }} \\
\text { counts }\end{array}$ & $\begin{array}{c}\text { Current } \\
(\mathrm{nA})\end{array}$ & $\begin{array}{c}i_{\text {spike }} \\
\text { counts }\end{array}$ & $\begin{array}{c}\text { Current } \\
(\mathrm{nA})\end{array}$ & $\begin{array}{c}i_{\text {spike }} \\
\text { counts }\end{array}$ \\
\hline 25 & 204 & 50 & 667 & 50 & 354 & 50 & 837 \\
75 & 36 & 150 & 53 & 150 & 12 & 150 & 55 \\
125 & 14 & 250 & 17 & 250 & 4 & 250 & 13 \\
175 & 4 & 350 & 6 & 350 & 0 & 350 & 1 \\
225 & 2 & 450 & 1 & 450 & 0 & 450 & 3 \\
275 & 3 & 550 & 1 & 550 & 1 & 550 & 1 \\
325 & 2 & 650 & 1 & 650 & 0 & 650 & 0 \\
375 & 2 & 750 & 0 & 750 & 1 & 750 & 0 \\
425 & 0 & 850 & 0 & & & 850 & 0 \\
475 & 2 & 950 & 0 & & & 950 & 0 \\
525 & 0 & 1050 & 0 & & & 1050 & 0 \\
575 & 1 & 1150 & 0 & & & 1150 & 0 \\
1450 & 1 & 1250 & 1 & & & 1250 & 0 \\
1550 & 0 & & & & & 1350 & 0 \\
1650 & 1 & & & & & & 1450 \\
\hline
\end{tabular}

Figure S4. Frequency counts vs. $i_{\text {spike }}$ obtained from CAs measured in different $E_{\text {ox }}$ 

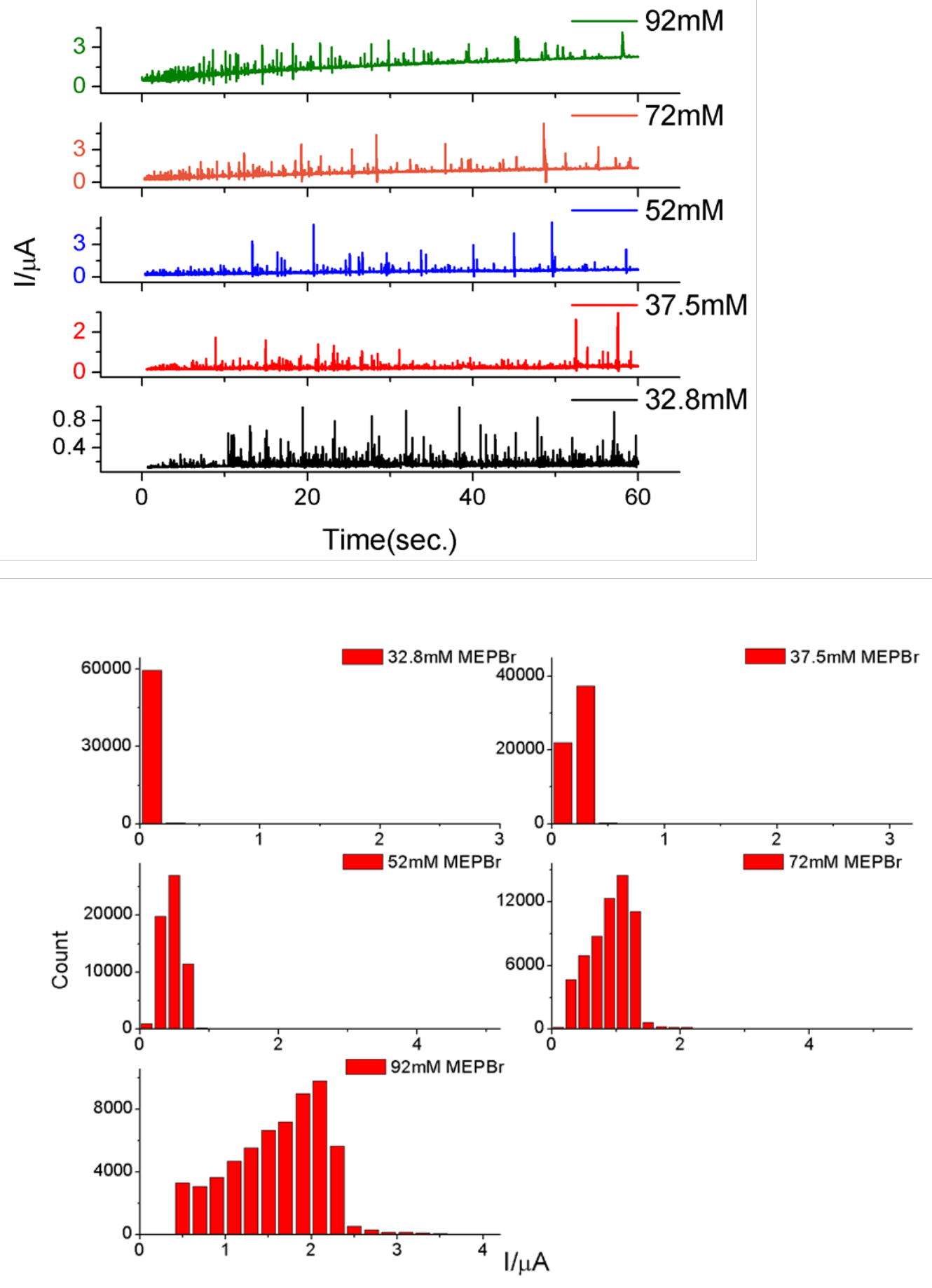

Figure S5. CAs measured in aqueous $\mathrm{MEPBr}$ solutions $\left(C_{M E P B r(a q)}=32.8,37.5,52,72,92 \mathrm{mM}\right)$ and frequency counts vs. $i_{\text {spike }}$ obtained from the CAs. 

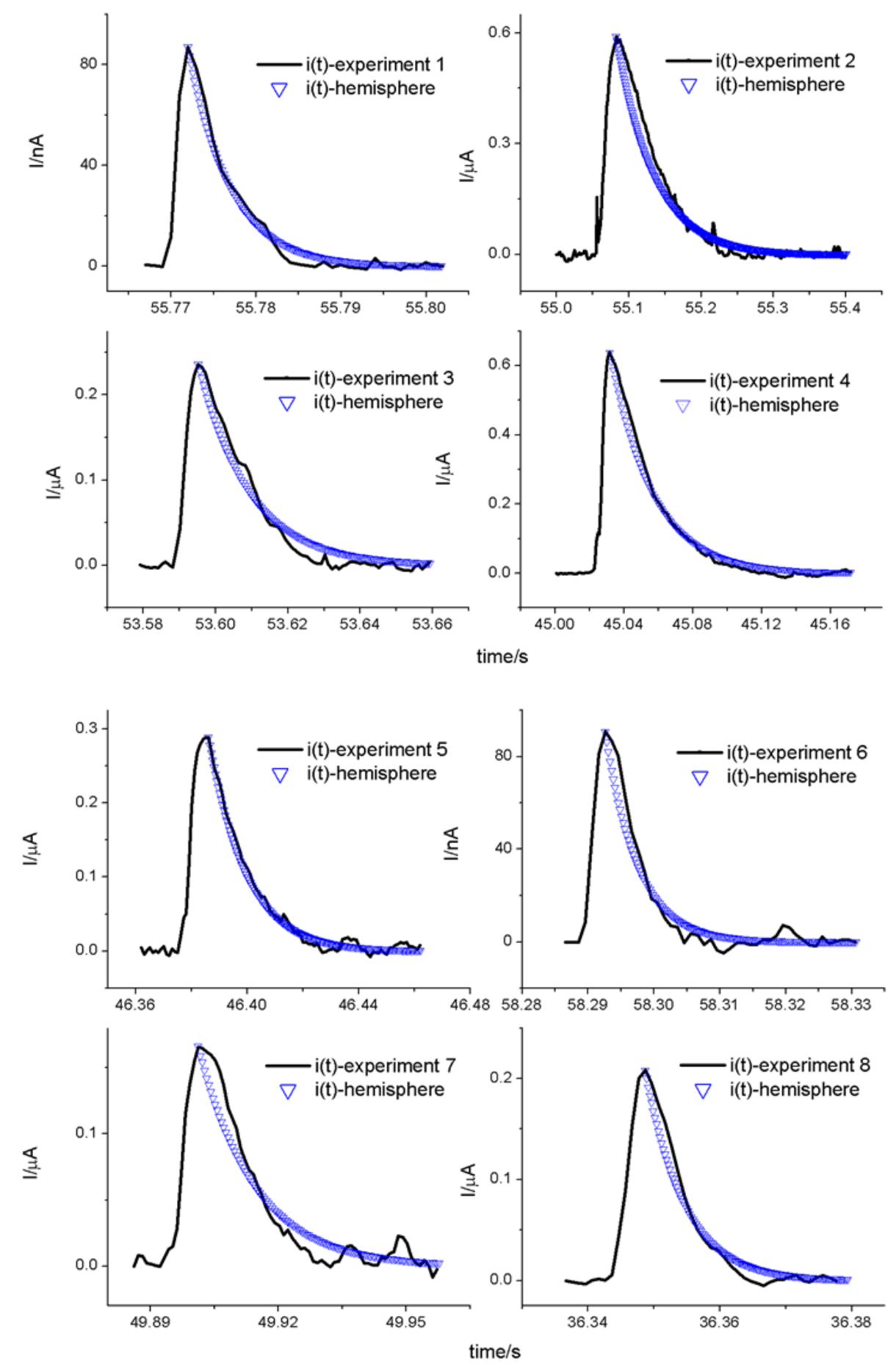

Figure S6. Eight different current spikes (black line) with different $i_{\text {spike }}$ attributed to the electrooxidation reaction of $\mathrm{Br}^{-}$in single $\mathrm{MEPBr}_{3}$ droplets from a CA in $C_{M E P B r(a q)}=72 \mathrm{mM}$, and their corresponding theoretical curves (blue triangle) derived from the bulk electrolysis model. 
Table S1. Estimated average $r_{\mathrm{e}}$ and $C_{B r^{-}}{ }_{\left(M E P B r_{3}\right)}$ by fitting current transients associated with $\mathrm{Br}^{-}$ electro-oxidation in single $\mathrm{MEPBr}_{3}$ droplets into the bulk electrolysis model shown in Figure S7.

\begin{tabular}{ccccc}
\hline $\begin{array}{c}\text { current } \\
\text { spikes }\end{array}$ & $r_{\mathrm{e}}(\mu \mathrm{m})$ & $\begin{array}{c}\text { Integrated charge } \\
(\mathrm{nC})\end{array}$ & $\mathrm{V}\left(\mu \mathrm{m}^{3}\right)$ & $C_{B r^{-}\left(\mathrm{MEPBr} r_{3}\right)}(\mathrm{M})$ \\
\hline 1 & 1.0 & 0.52 & 2.1 & 3.9 \\
2 & 3.3 & 42 & 72 & 9.1 \\
3 & 1.7 & 3.9 & 10 & 5.9 \\
4 & 2.3 & 19 & 24 & 12 \\
5 & 1.7 & 5.6 & 9.4 & 9.2 \\
6 & 1.0 & 0.64 & 2.1 & 4.7 \\
7 & 1.7 & 2.7 & 9.4 & 4.5 \\
8 & 1.1 & 1.9 & 2.8 & 11 \\
\hline average & $1.7( \pm 0.7)$ & 9.5 & 16 & $7.5( \pm 3.2)$ \\
\hline
\end{tabular}

